\title{
Comparative Study on Forecasting Model for Stock Index Future Price
}

\author{
Wenbo Wang*, He Nie \\ College of Economics, Jinan University, Guangzhou, China \\ Email: *marina2babe@163.com
}

How to cite this paper: Wang, W.B. and Nie, H. (2018) Comparative Study on Forecasting Model for Stock Index Future Price. Modern Economy, 9, 750-757. https://doi.org/10.4236/me.2018.94049

Received: March 21, 2018

Accepted: April 23, 2018

Published: April 26, 2018

Copyright $\odot 2018$ by authors and Scientific Research Publishing Inc. This work is licensed under the Creative Commons Attribution International License (CC BY 4.0).

http://creativecommons.org/licenses/by/4.0/

\begin{abstract}
The wavelet neural network has been widely applied in the forecasting field. However, due to its disadvantages of local minimal and slow convergence speed, its forecasting precision is limited in some extent. A forecasting model for stock index future price based on wavelet analysis and the improved PSO-based neural network would be put forward in this essay using the improved PSO-based algorithm to optimize the neural network. By using the Shanghai and Shenzhen 300 stock index futures' statistics as the samples, the sym 8 wavelet transformation techniques to denoise the statistics and then use the denoised stats to train and test the improved PSO-based neural networks. The study results show that the model in this essay could efficiently improve the forecasting quality of the stock index futures price.
\end{abstract}

\section{Keywords}

Forecasting Model, Stock Index Future Price, Wavelet Analysis, PSO, Neural Networks

\section{Introduction}

The stock index futures mean a kind of future with the stock index as the buying-and-selling basis. It's of great significance to carry out the forecasting for the stock index futures. It could not only avoid the systematic risk of the stock markets, but could also promote the rational fluctuation of the stock price [1]. We must scientifically analyze the stock market and try to forecast the stock index futures in multiple ways, and then precisely handle the uncertainty of investment profits in all factors in order to reduce the subjectivity and blindness in the process of investment decisioning and thesrefore avoid the risks.

As a non-linear dynamic system, the stock market possesses high complexity. Currently there are some effective methods for the forecasting of it, such as the 
neural networks [2] [3], the support vector machines method [4], the grey relation [5], the wavelet analysis [6] [7], the ARMA model [3], the GA-SVA model [1], the GJR-ARCH approach [8], the hyperbolic Gaussian model and the genetic networks [9] [10]. Among them, the neural network stands out as a mainstream method for its excellent non-linear approaching ability and self-learning and self-adapting abilities and plays an important role in the forecasting of the stock index futures [4].

The neural network is based on the minimal sample point error in the training process, and therefore the learning phenomena are inevitable and the restriction of the model's generalization ability would appear, which both greatly affect the forecasting quality [11]. Many scholars had made improvements on the method based on this shortcoming, Yang (2014) uses the wavelet analysis to pre-process the statistics and denoise them in order to reach better neural network training quality [2]. Li (2014) made an expansion on the neural network model [13]. Ma (2012) used the particle swarm to optimize the neural network [5]. Jiang (2013) combined the grey relation and the neural network to dynamically forecast the stock index [11].

Based on the problems appeared in the previous studies, this essay puts forward a forecasting model for stock index futures using the wavelet analysis and the improved PSO-based neural network. Firstly, we use the sym 8 wavelet transformation to denoise the chosen statistics, then optimize the neural network using the improved PSO-based neural network and use the denoised statistics to train and test. The study result shows that this model could effectively improve the forecasting results of the stock index futures, which proves the efficiency and practicality of this method.

The article is organized as follows. The first chapter is the introduction, which introduces the research background, the research situation at home and abroad, and puts forward the research contents and structure arrangement of the paper. The second chapter is the theoretical basis and model framework of the paper. The intelligent algorithm adopted in this paper is introduced in detail, and the algorithm is improved. The third chapter mainly describes the process of data analysis, the result of the model solution and the discussion of the results. First, the sym 8 wavelet transform is applied to denoise the selected data. The improved PSO neural network is used to optimize the neural network, and then trained and tested by noise reduction data pairs. The results show that the model proposed in this paper can effectively improve the prediction effect of stock index futures, and also shows the effectiveness and feasibility of the method. The fourth chapter is the summary of the paper and the prospect of the next step.

\section{Methodology}

\subsection{Wavelet Analysis}

The wavelet analysis is an effective method of joint time-frequency analysis. Different wavelets functions have different denoise effect on the financial statistics. 
For example Yang (2012) put forward the denoising requirements of the symN wavelet for the futures prices' time sequence in the orthogonal, the compact support and symmetry aspects, and repeatedly proved that the sym 8 has the best denoise effect [12]. Thus, we choose the sym8 wavelet as the denoise tool for the statistics.

\subsection{Improved PSO-Based Neural Network Model}

\subsubsection{Improved PSO Algorithm}

The PSO algorithm is a swarm intelligence based algorithm, using the swarm intelligent directed searching appeared in the swarm particles' cooperation and competition [5].

The basic formula for the PSO's particle optimization is as below [14]:

$$
\begin{gathered}
v_{i d}^{t+1}=w v_{i d}^{t}+c_{1} \times r_{1} \times\left(p_{i d}^{t}-x_{i d}^{t}\right)+c_{2} \times r_{2}\left(p_{g d}^{t}-x_{i d}^{t}\right) \\
x_{i d}^{t+1}=x_{i d}^{t}+v_{i d}^{t+1}
\end{gathered}
$$

where, $d=1,2, \ldots, n, I=1,2, \ldots, m, n$ stands for the dimensions of the searching space, $m$ stands for the number of population particles, $w$ stands for the inertia factor; $r_{1}$ and $r_{2}$ stands for the random numbers obeying the equidistribution in the interval of $(0,1), c_{1}$ and $c_{2}$ stands for the learning factor, $t$ stands for the iterations, $x_{i}^{n}$ stands for the space position of the particle $i$ in the $t$-th iteration, $v_{i}^{n}$ stands for the speed of the particle $i$ in the $t$-th iteration, $p_{i}^{t}$ and $p_{g}^{t}$ stands for the individual and global extreme value appeared in the particles' searching from the start to the current iteration.

In order to avoid the low searching precision and low iteration efficiency, we improve the PSO-based algorithm. We add in the mutation operator, which means after each update, if the random possibility is greater than 0.85 , we will randomly initialize the population, and otherwise we don't initialize it. The mutation operation widen the decreasing population search space in the iterations, making the particles able to jump out of the previous search into the optimized position, ensuring the variety of the population.

Meanwhile, we improved the PSO's inertia weight factor $\omega(k)$ in order to better balance the algorithm's searching ability, because this factor could influence the particles' speed and could attribute to either global search or local search based on whether it's greater or smaller.

$$
\omega(k)=\omega_{\max }-\frac{\left(\omega_{\max }-\omega_{\min }\right) \times k}{k_{\max }}
$$

where, $\omega_{\max }$ stands for the maximum of the inertia weight, is generally set as $0.8-0.9 ; \omega_{\min }$ stands for the minimum of the inertia weight, is generally set as 0.3 - 0.4; $k$ stands for the iterations; $k_{\min }$ stands for the maximum iterations.

\subsubsection{Optimized Neural Network Model}

This essay would choose the three-level BP neural network, where the input units respectively correspond to the stock indexes and forecasted stock indexes, 
the hidden-level neural units use the LSTF (Logarithmic sigmoid transfer function), and the neural units in the output-level use the pure linear transform function. The function for the evaluation of network training's average errors is as below:

$$
\text { SSE }=\frac{1}{2 p} \sum_{j=1}^{p} \sum_{i=1}^{n}\left(t_{i}-o_{i}\right)^{2}
$$

where, $o$ stands for the output of the network forecasting result, $t$ stands for the expected network output result, $S S E$ stands for the total neural network learning errors after the unification, $n$ stands for the unit number, $p$ stands for the sample number.

We would also combine the improved PSO and the BP algorithms based on the BP neural network model, using the improved PSO to search for the network's initial weight and threshold, then we use the BP neural network algorithm to train the improved PSO algorithm to find the optimized initial weight and threshold in order to find those of the model and eventually form the final model. This improved PSO-based neural network could overcome the shortcomings of low learning stability, low reliability and tendency of falling into local minimal problem, and also it provides better approaching quality and faster convergence speed.

\section{Empirical Analysis of Chinese Stock Market}

\subsection{Data Choosing and Preprocessing}

The Shanghai and Shenzhen 300 Stocks Index is basically used for China's stock index futures forecasting, its current month contract would be the most typical study object [12]. Thus, we choose the Index's current month contract from Dec

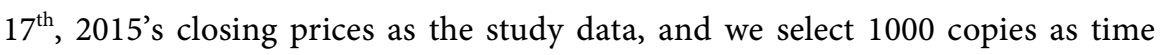
order sequence. Then we construct a $6 \times 1000$ matrix, with every line of 6 statistics as a group, making the first 900 groups of statistics as the model training samples, and the last 100 groups as the testing samples. Because of the different dimensions of the indexes, the removal of dimensions of the statistics is firstly done in order to compare the data, and also we must formalize the evaluation indexes and transform the range to get the result matrix $X=\left(x_{i j}\right)_{m \times n}$, which is

$$
x_{i j}=\frac{r_{i j}-\min _{i}\left\{r_{i j}\right\}}{\max _{i}\left\{r_{i j}\right\}-\min _{i}\left\{r_{i j}\right\}}, i=1,2, \ldots m ; j=1,2, \cdots, n
$$

where, $r_{i j}$ stands for the corresponding indexes' value, $x_{i j}$ stands for the value of no dimension and $x_{i j} \in[0,1], i=1,2, \cdots, m ; j=1,2, \cdots, n$.

\subsection{Forecast for Stock Index Futures}

We use the MATLAB programing to realize our goal in this section. First we use the neural network to roughly forecast the statistics after we unify all the data, with the BP neural network as the input with 5 input level nodes, 4 hidden level 
nodes, 1 output level node, 0.001 object error rate, 200 training steps. Use the pre-settlement price, the pre-closing price, the opening price, the highest price of a day as well as the lowest input price of a day as input training set and the closing price as the output test set. Then we use the sym8 wavelet as the statistics processing tools and the wavelet transformation to denoise the statistics. At last, we use the ordinary PSO-based neural network and the improved PSO-based neural network to respectively forecast the statistics and then compare the results.

We use the neural networks, the wavelet neural networks, the wavelet PSO-based neural network, and the wavelet improved PSO-based neural network to respectively make a forecast for statistics. We could see the results (portion) and comparison of the forecasting mean square error are in Table 1 and Table 2. The training graph of the wavelet improved QPSO-based neural networks is Figure 1, and the comparisons of the forecasting results (10 days) are in Figure 2.

\subsection{Discussion}

As we could see in Figure 1, compared with PSO-based neural network training curve, the improved PSO-based neural network needs about 40 iterations to realize the goal with fast convergence speed and not easily fall into local optimum. Also in Figure 2 and Table 1, the improved PSO-based neural network

Table 1. Comparison of the forecasting results (portion).

\begin{tabular}{ccccccccc}
\hline & \multicolumn{2}{c}{ BP } & \multicolumn{2}{c}{ W-BP } & W-PSO-BP & W-improved PSO-BP \\
\cline { 2 - 8 } Actual value & Forecasting & Errors & Forecasting & Errors & Forecasting & Errors & Forecasting & Errors \\
\hline 2464.2 & 2517.99 & $2.18 \%$ & 2504.53 & $1.64 \%$ & 2477.95 & $0.56 \%$ & 2487.37 & $0.94 \%$ \\
2504.4 & 2549.45 & $1.80 \%$ & 2497.90 & $0.26 \%$ & 2471.06 & $1.33 \%$ & 2480.05 & $0.97 \%$ \\
2509 & 2531.36 & $0.89 \%$ & 2489.75 & $0.77 \%$ & 2463.80 & $1.80 \%$ & 2472.13 & $1.47 \%$ \\
2487.2 & 2496.36 & $0.37 \%$ & 2479.96 & $0.29 \%$ & 2455.93 & $1.26 \%$ & 2463.45 & $0.95 \%$ \\
2439.8 & 2500.78 & $2.50 \%$ & 2470.99 & $1.28 \%$ & 2448.34 & $0.35 \%$ & 2455.11 & $0.63 \%$ \\
2476 & 2504.11 & $1.14 \%$ & 2463.40 & $0.51 \%$ & 2441.76 & $1.38 \%$ & 2447.49 & $1.15 \%$ \\
2469.2 & 2531.42 & $2.52 \%$ & 2457.71 & $0.47 \%$ & 2436.40 & $1.33 \%$ & 2440.83 & $1.15 \%$ \\
2498.2 & 2512.36 & $0.57 \%$ & 2458.31 & $1.60 \%$ & 2431.68 & $2.66 \%$ & 2486.53 & $0.50 \%$ \\
2470.2 & 2488.21 & $0.73 \%$ & 2455.62 & $0.59 \%$ & 2424.24 & $1.86 \%$ & 2430.21 & $1.62 \%$ \\
2427.4 & 2498.52 & $2.93 \%$ & 2436.99 & $0.40 \%$ & 2418.42 & $0.37 \%$ & 2418.51 & $0.37 \%$ \\
\hline
\end{tabular}

Table 2. Comparison of the forecasting mean square error.

\begin{tabular}{ccccc}
\hline & BP & W-BP & W-PSO-BP & W-improved PSO-BP \\
\hline Mean Square Error & 1919.36 & 1871.92 & 1960.08 & 1607.47 \\
\hline
\end{tabular}




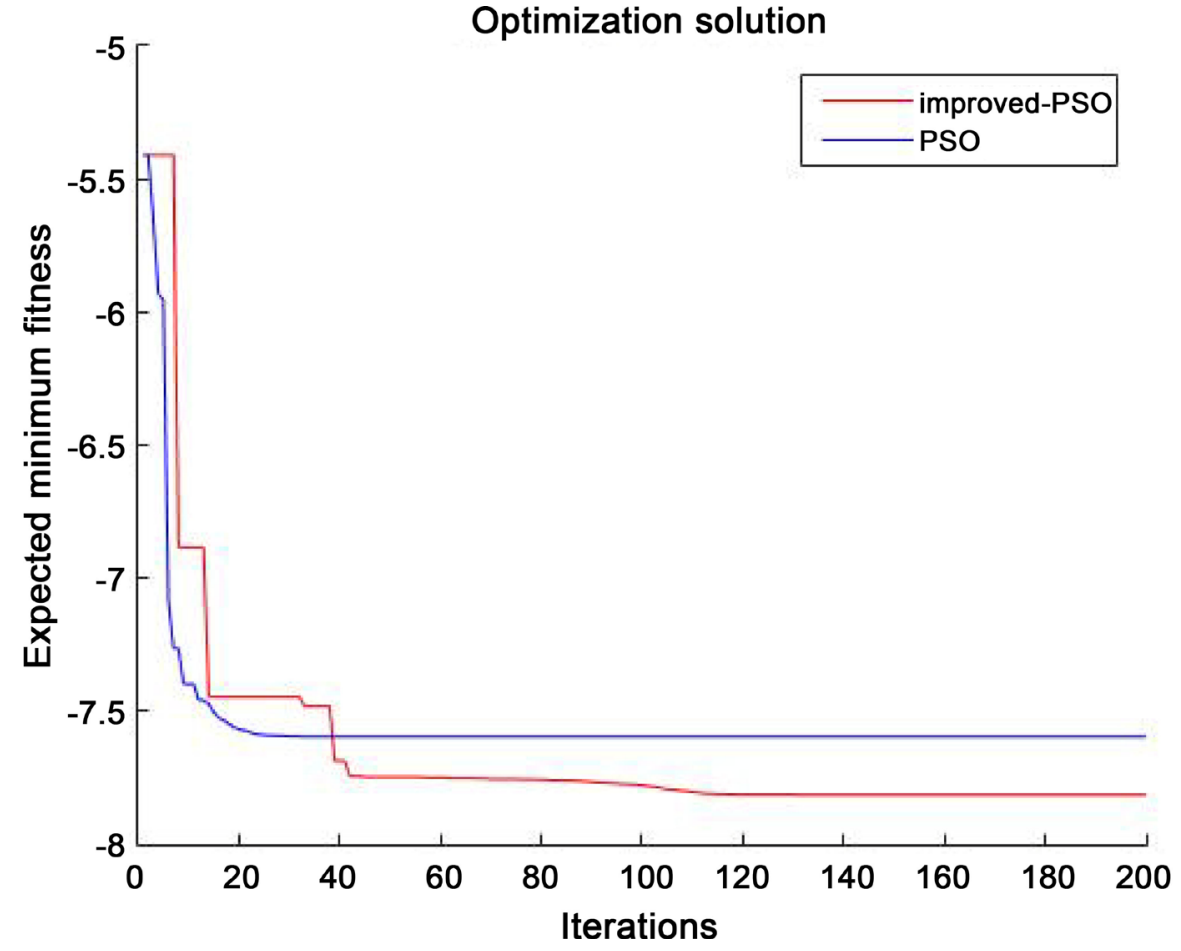

Figure 1. Comparison of improved-PSO and PSO-based neural network training graph.

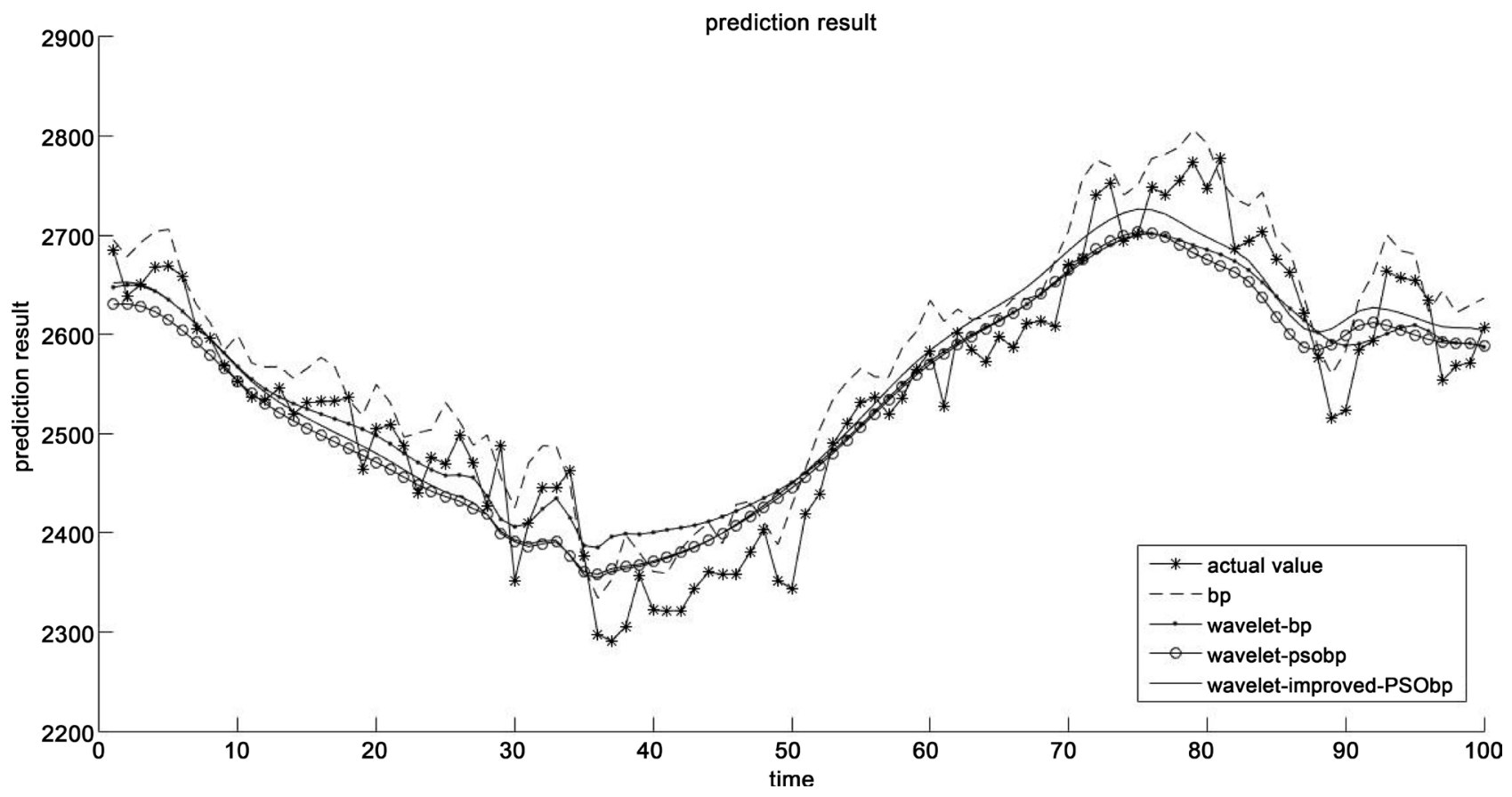

Figure 2. Comparison of the forecasting results.

has better forecasting results than other methods and closer to the actual value. We could also see that the improved PSO-based neural network has the least forecasting mean square errors in Table 2, and the forecasting results can be comparatively accepted. Therefore these results could prove that this model posses practicality and precision. 


\section{Conclusions}

We put forward a forecasting model for stock index future price based on wavelet analysis and the improved PSO-based neural network, which could not only effectively denoise the statistics of the indexes, and could also improve the learning ability, avoid local optimization and faster the convergence speed of the BP neural networks using the improved PSO-based algorithm. Last but not the least, our experiments show that the model in this essay could well lower the error rate of the forecasting.

The main contributions of this paper are the model proposed in this paper that can effectively improve the prediction effect of stock index futures. In this study, the technique of wavelet analysis is applied to the original data. The technology of wavelet decomposition and reconstruction is used to extract the stock price trend hidden in the noise. In this paper, the PSO algorithm is optimized and PSO is used to optimize the neural network. The innovation of this paper is the application of the noise reduction financial data, and finally makes the price prediction.

In this paper, a prediction model of stock index futures based on wavelet analysis and improved PSO optimization neural network is greatly improved for the prediction of stock index futures. Compared with the existing models, the network model proposed in this paper has higher prediction accuracy, but the prediction stability and convergence speed are relatively poor, which is also a problem that we need to further solve in this research. Due to the time and length limitation of the thesis, there are still some aspects to be improved.

1) Although this paper aiming at shortcomings of the existing prediction model proposes a new model, the performance of BP neural network was improved, and the prediction accuracy was also improved, but the improved algorithm because some parameters are difficult to determine, there are poor stability problems in future research in this optimization problem.

2) Financial data types are complicated, this paper only selects the Shanghai and Shenzhen 300 stock index futures contract month from the date of listing to November 17, 2015 closing price time series as the research data, the research data is relatively small, but also does not take into account the problems such as periodic data, in the future research should consider the problem of periodic financial data which can be used at the same time, technical indicators to predict more optimized considering the story.

\section{Acknowledgements}

The authors acknowledge the financial support of this research by Natural Science Foundation of China under Grant 61201458.

\section{References}

[1] Li, J. (2011) Research on Stock Index Future Price Forecasting Methods Based on Wavelet Transform and GA-SVR Model. South China University of Technology, 
Guangzhou.

[2] Yang, C., Liu, X.H., Economics C.O., et al. (2014) Forecasting Model for Stock Index Future Price Based on Wavelet Analysis and BP Neural Network. Journal of Qingdao University, 1, 101-105.

[3] Xie, C., Zhu, Z. and Yu, C. (2012) A Study of Feedback Trading in Stock Index Futures: An Empirical Analysis on Asian Markets. IEEE 5 th International Conference on Advanced Computational Intelligence, Vol. 8267, 900-902.

[4] Sai, Y., Zhang, F.T. and Zhang, T. (2013) Research of Chinese Stock Index Futures Regression Prediction Based on Support Vector Machines. Chinese Journal of Management Science, 3, 35-39.

[5] Ma, J.M., Xu, Z.R. and Wang, B.Z. (2012) A PSO-Based Combined Forecasting Grey Neural Network Model. Computer Engineering \& Science, 2, 146-149.

[6] Liu, X.L., Wang, X.P. and Finance, S.O. (2015) Research on High Frequency Data Forecasting of Stock Index Futures Market Based on Wavelet Analysis. Systems Engineering-Theory \& Practice, 6, 1425-1432.

[7] Liu, J., Zhang, Y. and Li, S.Y. (2011) The Research of Stock Index Futures Margin Setting Model Based on Wavelet Analysis Method and Technology. 4th International Conference on Business Intelligence and Financial Engineering, 17-18 October 2011, Wuhan, 447-451. https://doi.org/10.1109/BIFE.2011.137

[8] Wang, Y.H. (2009) Nonlinear Neural Network Forecasting Model for Stock Index Option Price: Hybrid GJR-GARCH Approach. Expert Systems with Applications, 36, 564-570. https://doi.org/10.1016/j.eswa.2007.09.056

[9] Bäuerle, N. and Pfeiffer, R. (2013) A Joint Stock and Bond Market Based on the Hyperbolic Gaussian Model. European Actuarial Journal, 3, 229-248. https://doi.org/10.1007/s13385-012-0060-6

[10] Chen, Y. and Wang, X.C. (2015) A Study on High-Frequency Futures Trading Strategy Based on Variable Selection and Genetic Network Programming. Chinese Journal of Management Science, 10, 47-56.

[11] Jiang, H. and Gui, X.U. (2013) Dynamic Prediction of Stock Index Based on Grey System and BP Neural Network. Mathematics in Practice \& Theory, 22, 28-37.

[12] Sun, H.T., Yang, D.P. and Cong, L.I. (2012) Prediction of Stock Index Future Price Based on BP Neural Network. Journal of Qingdao University, 3, 93-96.

[13] Li, X.Z. and Meng, Z.Q. (2014) Study of Extenics Neural Networks Model and Stock Index Futures Analysis. Computer Science, S2, 440-446.

[14] Cheng, S.F., Cheng, X.H. and Lu, Y. (2014) Application of Wavelet Neural Network with Improved Particle Swarm Optimization Algorithm in Power Transformer Fault Diagnosis. Dianli Xitong Baohu Yu KongzhilPower System Protection \& Control, 42, 37-42. 